\title{
Téoros
}

Revue de recherche en tourisme

\section{Par-delà le tourisme}

Parcs Nunavilk: un outil pour inscrire la culture inuite dans le global

\section{Thibault Martin}

Volume 31, numéro 1, 2012

Parcs du Nunavik

URI : https://id.erudit.org/iderudit/1020704ar

DOI : https://doi.org/10.7202/1020704ar

Aller au sommaire du numéro

\section{Éditeur(s)}

Université du Québec à Montréal

ISSN

0712-8657 (imprimé)

1923-2705 (numérique)

Découvrir la revue

Citer ce document

Martin, T. (2012). Par-delà le tourisme : parcs Nunavik : un outil pour inscrire la culture inuite dans le global. Téoros, 31(1), 3-8.

https://doi.org/10.7202/1020704ar

Ce document est protégé par la loi sur le droit d'auteur. L’utilisation des services d’Érudit (y compris la reproduction) est assujettie à sa politique d'utilisation que vous pouvez consulter en ligne.

https://apropos.erudit.org/fr/usagers/politique-dutilisation/ 


\section{Par-delà le tourisme Parcs Nunavik : un outil pour inscrire la culture inuite dans le global}

Thibault MARTIN, Ph.D.

Professeur au Département des sciences sociales

Titulaire de la Chaire de recherche du Canada sur la gouvernance autochtone du territoire

Université du Québec en Outaouais (UQO)

thibault.martin@uqo.ca

Par une journée de l'hiver 1996, alors que j'effectuais, dans le cadre de ma thèse de doctorat, un terrain de recherche à Umiujaq, mon interprète décida de m'emmener au bord du lac Guillaume-Delisle. Après un long moment de contemplation, il rompit le silence pour me dire: «Un jour le gouvernement va créer ici un parc. Il y a des Inuits qui sont contre, car ils ont peur qu'on ne puisse plus aller chasser à cause des touristes et des environnementalistes [...] Mais moi je n'ai pas peur : emmenez-en, des touristes! Nous sommes prêts à les accueillir.» Bien que ma thèse ne portât pas sur les aires protégées, cette déclaration enthousiaste de mon interprète, généralement assez réservé, m'avait surpris, si bien que je l'avais prise en note, sans vraiment savoir quel usage j'en ferais.

Le 14 décembre 2012, 16 ans plus tard, le gouvernement du Québec annonçait la création du parc national Tursujuq, celui-là même dont rêvait mon interprète. Avec ses 26900 km², il est le plus grand parc de la province du Québec. Pourtant, selon le projet original, il n'aurait dû couvrir que $15000 \mathrm{~km}^{2}$; c'est sous la pression des Inuits que le gouvernement provincial a accepté d'étendre les limites du parc pour y inclure le bassin de la Nastapoka que les Inuits souhaitaient mettre à l'abri des développements hydroélectriques planifiés par Hydro-Québec.

S'il y a 20 ans la création d'un parc préoccupait beaucoup d'Inuits, aujourd'hui les choses ont bien changé. En effet, avec la signature, en 2002, de l'Entente de partenariat sur le développement économique et communautaire au Nunavik (connue sous le nom d'entente Sanarrutik), l'Administration régionale Kativik (ARK) a obtenu le mandat de coordonner le développement des projets de parcs nationaux du Nunavik et d'assurer leur gestion après leur création. Le parc national Tursujuq est le troisième des parcs nationaux créés au Nunavik, et deux autres sont planifiés. Le développement et la gestion de ces parcs sont effectués par les Inuits dont les choix de gestion sont orientés par des considérations socioculturelles et pas seulement en fonction d'objectifs de protection environnementale. On peut donc mesurer le chemin parcouru par les Inuits au cours des dernières années; ceux-ci, en plus de faire respecter leur droit de pratiquer leurs activités de chasse, de pêche et de piégeage, inscrit dans la Convention de la Baie-James, ont aussi gagné la possibilité de définir les aires à protéger et la manière d'effectuer cette protection. Ils peuvent également orienter les activités, notamment touristiques, mais aussi scientifiques, qui s'y déroulent.

Néanmoins, certaines choses n'ont pas changé depuis la déclaration de mon interprète, en particulier la manière ambiguë avec laquelle les Inuits abordent la question du tourisme. En effet, certains craignent encore que les activités touristiques associées aux parcs ne viennent entraver leurs propres activités sur le territoire, tel que le recommande un participant lors des audiences publiques tenues en vue de la création du parc national Tursujuq : «[Il faut] s'assurer que les touristes qui vont sur le territoire ne nuisent pas à nos activités» (MDDEP, 2008a : lignes 1524-1525). De même, lors des audiences publiques tenues dans le cadre de la création du parc national Kuururjuaq, 6 intervenants sur les 22 résidents de Kangiqsualujjuaq qui y ont participé ont manifesté leurs inquiétudes concernant la poursuite des activités traditionnelles (MDDEP, 2007a : 8). Toutefois, les Inuits et les autorités inuites, en particulier l'Administration régionale Kativik et Makivik, considèrent le tourisme comme un moyen de créer des emplois culturellement pertinents et qui s'inscrivent dans une perspective de développement durable (KRG, 2007).

En tant que revue consacrée au tourisme, Téoros nous est apparu comme le médium idéal pour présenter un numéro thématique consacré à Parcs Nunavik. En effet, la question des parcs nationaux est intimement liée à celle du tourisme puisqu'un des mandats des organismes gestionnaires des parcs est d'y favoriser la pratique d'activités de plein air, mais aussi parce qu'aujourd'hui les Autochtones veulent s'appuyer sur le tourisme pour favoriser le développement économique de leurs communautés (Antomarchi, 2010, 2009; D'Amours, 2011; Lemelin et al. 2012; Lequin et Cloquet, 2006; Tulugak et Murdoch, 2007). À cet égard, l'Administration régionale Kativik (ARK) publiait récemment un document d'orientation sur le tourisme dans lequel elle affirme que "[t]he development of an authentic cultural, eco-friendly, and sustainable 
tourism industry will have a number of positive effects, including community development, youth employment and cultural reinforcement» (KRG, 2009: 2).

Toutefois, cet énoncé de l'ARK révèle un élément important du rôle que les Inuits veulent faire jouer au tourisme. Pour eux, celui-ci ne doit pas avoir pour seul objectif la création d'emplois, mais doit aussi contribuer à la pérennisation de la culture inuite. Certes, les entreprises touristiques conventionnelles, notamment les pourvoiries et les compagnies de croisières, cherchent avant tout à créer des emplois et à générer des revenus monétaires. Néanmoins, l'entrepreneuriat touristique commence à s'intéresser aux activités de tourisme culturel (voir par exemple la course de traîneaux à chiens Evakak, l'Inuit Odyssey ou le Aqpik Jam Festival). De plus, à Puvirnituq, une entreprise touristique, le Centre de formation du Nunavik en survie arctique, accueille chaque année une cinquantaine de touristes, essentiellement des Européens, qui viennent s'initier à la culture inuite (Antomarchi, 2009 : 54). Ajoutons à cela le développement, toujours à Puvirnituq, d'un important projet de complexe mixte touristique et culturel qui doit combiner un musée, un centre de transmission culturelle (ateliers de sculpture et d'estampes) et un centre de communication (Internet, radio). Le nom de ce complexe pourrait être «Saputik Museum (ce que la mer transporte et laisse : notion d'héritage)» (Antomarchi, 2009: 53). Surtout, les activités proposées dans les parcs ne sont pas tant présentées comme des activités économiques, mais comme des acteurs du « renforcement culturel ». Une étude que nous avons faite des plans directeurs des parcs canadiens du circumpolaire (Martin, à paraître) montre que les références aux activités touristiques en tant que source de développement économique, sans être inexistantes, y sont rares et que le tourisme ne constitue généralement qu'un élément secondaire des objectifs des plans directeurs.

Cet intérêt modéré pour la question du tourisme au sein des parcs - en dehors de sa capacité à renforcer la culture inuite - peut s'expliquer de plusieurs manières. Tout d'abord, les Inuits ont conscience que le potentiel d'attraction des parcs canadiens du circumpolaire est assez limité, comparativement aux autres activités touristiques (croisières, aventures, observation de la nature, chasse et pêche) qui attirent chaque année plusieurs centaines de milliers de visiteurs (Grenier, 2007 : 55). Au Nunavik, les acteurs du développement touristique savent que leur territoire est pour l'instant une destination relativement peu connue, que le coût des transports, les infrastructures déficientes, le manque de main-d'œuvre qualifiée (Makivik, 2012 : 407) et l'accessibilité parfois difficile des parcs ne permettront pas, à tout le moins dans un avenir proche, de tabler sur un nombre de visiteurs important (Lemelin et al., 2012). Ce sont autant d'obstacles structurels qui limitent la fréquentation et, donc, les retombées économiques des parcs.

Cependant, cet intérêt modéré pour le tourisme au sein des parcs s'explique surtout, selon notre analyse, par le fait que, pour les Inuits, la raison d'être des parcs se situe ailleurs que dans les activités économiques. Il s'agit d'abord de protéger le territoire et la culture inuits. Les parcs doivent être un moyen de «vivre et [d']enseigner le mode de vie traditionnel» (Parc national du Canada Vuntut, 2010 : vii-vii). Ils doivent permettre de «façonner l'avenir grâce au passé» (Parc national du
Canada Vuntut, 2010 : vii-vii). Il s'agit aussi pour les Inuits de s'appuyer sur les parcs pour faire découvrir et partager leur mode de vie avec les non-Autochtones, tel que l'énoncé de Parcs Nunavik l'indique : «Parcs Nunavik se consacre à la protection $[\ldots]$ Cette protection est axée sur la conservation par l'entremise d'activités éducatives et récréatives destinées aux Nunavimmiut et aux voyageurs du monde entier» (Parcs Nunavik, 2012).

Ce projet d'utiliser les parcs et les activités pratiquées par les touristes comme médiums pour rejoindre «le monde» apparaît également dans plusieurs parcs nationaux déjà implantés dans d'autres territoires autochtones. Ainsi le Conseil de cogestion du parc national du Canada des Monts-Torngat écrivait, à l'occasion de la publication de son plan directeur, que celui-ci «répond aux désirs des Inuits du Nunatsiavut et du Nunavik qui souhaitent renouer avec les monts Torngat, transmettre le savoir des aînés aux futures générations inuites, protéger ce territoire à jamais et partager l'histoire inuite du Tongait KakKasuangita SilaKijapvinga avec les générations canadiennes à venir» (Parc national du Canada des MontsTorngat $2010: \mathrm{v}$ ).

Le plan directeur du parc Quttinirpaaq, quant à lui, stipule qu'un des objectifs du parc est de faire en sorte que les visiteurs «puissent jouir de la relation particulière que les Inuits entretiennent avec le territoire» (Parc national du Canada Quttinirpaaq, 2009:3).

En somme, lorsque mon interprète parlait d' «accueillir» les touristes, il n'entendait pas les «héberger», mais plutôt leur faire partager la culture inuite et leur ouvrir ainsi leur intimité. Si à l'époque je ne percevais pas le véritable sens de cet «accueil», aujourd'hui les exemples des différents parcs établis dans le Nord canadien révèlent que le désir de recevoir des touristes est davantage perçu comme un instrument servant à inscrire la culture inuite dans le global que comme le moyen de favoriser le développement économique. Notre analyse des plans directeurs des différents parcs du circumpolaire révèle que les Autochtones souhaitent avant tout que les parcs contribuent à l'actualisation de leur culture et de leur relation à la nature. Ces parcs doivent à ce titre permettre la transmission des savoirs entre les générations, encourager le maintien des activités de chasse, de pêche et de rassemblement sur les territoires ancestraux, ainsi que l'usage des langues autochtones, etc. (Martin, à paraître). Pour atteindre cet objectif, les Inuits souhaitent, par l'intermédiaire des activités touristiques, partager leur mode de vie avec les autres Canadiens, voire le reste du monde, afin d'obtenir une reconnaissance, tel que l'indique ici ces propos : «En échange des beautés et des richesses de notre culture, nous pouvons obtenir non seulement de l'argent, mais aussi une reconnaissance de la part d'autres cultures» (Tulugak et Murdoch, 2007, cité dans Antomarchi, 2009 : 57). Il s'agit donc, pour les Inuits, de justifier qu'on leur laisse l'espace nécessaire, non seulement géographique, mais aussi politique, pour que leur culture puisse s'épanouir et s'actualiser. On entend par là se perpétuer en évoluant et non pas simplement en se reproduisant à l'identique, comme l'indique le plan directeur du parc national du Canada des Monts-Torngats : «[R]aconter l'histoire des Inuits, accroître la compréhension des Canadiens quant au lien particulier qui unit les Inuits au riche patrimoine 
culturel $[\ldots]$ faciliter $[\ldots]$ les rassemblements contemporains qui célèbrent le parc en tant que territoire inuit » (Parc national du Canada des Monts-Torngat, $2010: \mathrm{x}$ ).

La même chose peut être dite des parcs du réseau de Parcs Nunavik, bien qu'il soit difficile de comparer la documentation. En effet, les plans directeurs des parcs du réseau de Parcs Canada sont rédigés directement par chacun des conseils de gestion, sur lesquels siègent des représentants autochtones (dans le cas du parc national du Canada des Monts-Torngat, tous les membres du conseil de cogestion sont des Inuits), ce qui permet d'accéder à la vision spécifique que les acteurs autochtones ont du rôle que doit jouer un parc au sein de leur projet de société. En revanche, les plans directeurs des parcs du Nunavik sont rédigés par les employés du ministère du Développement durable, de l'Environnement et des Parcs (MDDEP), ce qui, malgré le fait qu'ils soient le résultat de consultations importantes et qu'ils soient destinés à être mis en œuvre par Parcs Nunavik, donne une couleur différente, plus technique et plus uniforme. Néanmoins, on y retrouve le même objectif culturel, bien qu'il se cache parfois derrière l'expression «ethnotourisme» (MDDEP, 2005 : 35). Ainsi les plans directeurs des trois premiers parcs du Nunavik affirment que «les activités doivent mettre en valeur la culture inuite» (MDDEP, $2005: 35$ ), que le parc «offre l'occasion de prendre contact avec la culture inuite» (MDDEP, 2005 : 42). La mise en valeur du parc national Tursujuq doit ainsi montrer que «[l]es liens qui unissent les Inuits et les Cris à la nature constituent une dimension importante de la réalité de la région» (MDDEP, 2008b : 29) et que «[1]'offre éducative mettra en valeur non seulement les cultures inuite et crie, mais fera également ressortir comment ces deux nations ont su cohabiter sur le territoire» (MDDEP, 2008b : 33). En ce qui concerne le parc Kuururjuaq, «[1]'offre éducative mettra en valeur la culture inuite et la richesse des contes et légendes de la péninsule Ungava-Labrador» (MDDEP, 2007b : 30).

En somme, les parcs constituent pour les Inuits une institution centrale dans la production de leur société ainsi que dans l'affirmation de leur identité, voire de leur "nationalisme» - dans le sens de construction de la nation. Ce constat n'est pas sans rappeler les travaux de Nash (1967) qui mettent en évidence que la création des parcs "nationaux» est une des constituantes, notamment aux États-Unis et au Canada, de la construction de l'identité nationale. À cet égard, il est intéressant de noter l'évolution de la relation qui s'opère actuellement entre les Inuits du Nunavik et le Québec puisque l'institution nationale, «Parcs Québec», délègue la gestion des parcs nationaux du Québec nordique à une institution, «Parcs Nunavik», qui affiche son engagement dans la défense du territoire et de la culture inuits. Il s'agit là d'une manifestation de la nouvelle relation de "nation à nation" que veut établir le Québec avec les Autochtones, à tout le moins ceux qui sont conventionnés. En accordant aux Inuits la possibilité de créer leur propre institution de gestion des parcs, «Parcs Nunavik», Québec leur permet de progresser dans la création d'institutions «nationales». Certes, puisqu'ils sont en territoire québécois, ces parcs restent des parcs nationaux du Québec, même si le mot québécois est masqué dans l'intitulé du parc. Il s'agit néanmoins d'une avancée symbolique puisque, dans le cas des autres parcs canadiens, ceux-ci sont toujours dénommés «parcs nationaux du Canada", même lorsqu'ils sont établis en territoire autochtone (incluant au Nunavut). Cela rejoint la thèse de Kaufmann et Zimmer (1998) qui postulent que la valeur discursive des espaces naturels permet un usage politique des parcs nationaux. On comprendra, face à cet usage sociétal (pérenniser la culture), identitaire (se doter d'une institution nationale) et politique (affirmer la souveraineté inuite des territoires), que le tourisme, qui n'apparaît que comme une simple activité économique, n'a pas de raison d'occuper une place centrale dans l’argumentaire développé sur les parcs.

Néanmoins, comme le tourisme a été identifié «as a major driver for economic growth by regional stakeholders» (KRG, 2009 : 2), il est donc fort probable que celui-ci prenne de plus en plus de place au sein des parcs du Nunavik. Cela est d'autant plus vrai que le secteur touristique traditionnel (chasse et pêche ainsi que croisière) fait actuellement face à des difficultés importantes et de diverses natures (Lemelin et al., 2012). Cette montée en importance du tourisme dans les parcs se manifeste déjà à travers différentes initiatives, telle que l'Inuit Odyssey qui voit une entreprise de tourisme conventionnelle s'arrimer à un parc pour offrir un produit touristique à la fois d'aventure et culturel. Ce produit touristique connait un certain succès et a même reçu les éloges du National Geographic Traveler (Lemelin et al., 2012). Toutefois, compte tenu de la prudence habituelle des Inuits et de leur tradition de consultation au niveau communautaire (Larivière, 2010), ainsi que de leur réticence face au tourisme si celui-ci repose sur des impératifs commerciaux plutôt que communautaires (Lequin, 2003), il est probable qu'ils ne se précipitent pas dans cette direction sans en avoir évalué les conséquences sur leurs autres priorités, notamment culturelle et environnementale. C'est pourquoi il est difficile pour l'instant de débattre du tourisme au sein des parcs sans le mettre en relation avec les autres éléments constitutifs de la socialisation du territoire. Malgré tout, il nous a semblé important de présenter les différents facteurs qui vont contribuer à orienter la manière dont les Inuits vont «travailler» de l'intérieur cet enjeu du tourisme au sein des parcs. Ce numéro thématique de Téoros se propose donc d'ouvrir le débat sur ces facteurs. Les différents articles présentés vont des conditions politiques structurant la gouvernance des parcs aux premières initiatives touristiques en passant par la patrimonialisation du territoire, sans compter la question des changements climatiques.

Le dossier débute par l'article d'Alain Hébert, «Les parcs nationaux du Nunavik et du territoire d'Eeyou Istchee-BaieJames : un nouveau modèle de gouvernance des "parcs habités" ». Ce texte opère un retour sur la création de Parcs Nunavik. Il situe celle-ci dans le cadre plus large du processus de renouveau de la gouvernance des parcs entamé par le gouvernement du Québec en 2002, à la suite de la modification de la Loi sur les parcs (2001). Dans cette perspective, il analyse les différents modèles québécois de gouvernance des parcs : ceux situés en territoire autochtone conventionné (Nunavik et Eeyou IstcheeBaie-James) et ceux au sud du $50^{\mathrm{e}}$ parallèle, gérés par la Société des établissements de plein air du Québec (Sépaq). Il expose les différences à la fois entre le mode de gouvernance autochtone et celui mis en place par la Sépaq, mais aussi entre les modèles autochtones eux-mêmes. Il en conclut que ces différences 
attestent du travail réflexif que les Autochtones effectuent pour mettre en place des modèles de gestion adaptés à leurs réalités spécifiques, notamment sociales. Il met aussi en perspective ce qui se donne à voir au Nunavik avec le processus global d'intégration de plus en plus important des acteurs locaux dans la gouvernance des aires protégées.

L'article de Sébastien Grammond, Caroline Beaudry et Guy Chiasson, «La contribution du droit à la gouvernance territoriale : le cas du projet de parc national Tursujuq", suit. Il offre une perspective d'un point de vue juridique et politique du processus de création du parc national Tursujuq. L'article se base sur une étude des positions exprimées lors des audiences publiques tenues avant la création du parc. Leur analyse met en évidence l'importance pour les Inuits et les Cris de la Convention de la Baie-James, qui leur garantit le droit de pratiquer leurs activités traditionnelles. Elle montre aussi comment les Inuits ont occupé l'espace qui leur était offert par les audiences pour demander que le bassin de la rivière Nastapoka soit inclus dans les limites du parc, bien que cette dernière ait été identifiée dans l'entente Sanarrutik (2002) comme une des rivières d'intérêt pour Hydro-Québec. D'ailleurs, dans le plan directeur provisoire du parc, le bassin de la Nastapoka avait été exclu par le MDDEP. Toutefois, en décembre 2012, le gouvernement québécois s'est rallié à la position des Inuits et a élargi les limites du parc pour y inclure le bassin de la Nastapoka. Cet article nous permet de mieux comprendre la dynamique de gouvernance au Nunavik puisqu'il met en évidence comment les Autochtones ont tiré profit des mécanismes de gouvernance conjointe du territoire pour amener le gouvernement à prendre une décision qui respectait davantage leurs droits et leurs intérêts.Toujours dans le domaine de la gouvernance, «L'aire protégée transfrontalière Torngat-Koroc : gouvernance, tourisme et retombées économiques potentielles dans les territoires du Nunatsiavut (Labrador) et Nunavik (Québec)» — un texte de Raynald Lemelin - s'intéresse à la création, plus ou moins en parallèle, du parc national du Canada des MontsTorngat (dans la province de Terre-Neuve-et-Labrador) et du parc national Kuururjuaq (au Nunavik). Ces deux parcs sont contigus et ont pour objectif de protéger chacun une partie d'un territoire qui constitue une unité géographique. La juxtaposition de ces deux parcs nationaux, l'un sous juridiction fédérale et l'autre québécoise, constitue une aire protégée transfrontalière. L'établissement de ce type d'aire protégée représente de nombreux défis, mais constitue simultanément une occasion de faire des avancés en termes de gouvernance. C'est ce qui, selon l'étude de l'auteur, serait en train de se produire puisque la collaboration entre les deux structures de gestion s'avère efficace et produit des retombées économiques réelles, notamment en stimulant les activités touristiques. Cela dit, le processus qui a conduit à ce résultat s'est avéré long, complexe et parsemé d'embûches. L'auteur conclut en suggérant que le succès final repose sur l'implication des Inuits dans la création des deux parcs et sur la reconnaissance par les différents gouvernements de la nécessité d'établir une gestion coopérative incluant sur un pied d'égalité les populations autochtones.

L'article de Julie Bibaud, qui, lui aussi, est basé sur une analyse de discours, s'intéresse à la «Patrimonialisation des territoires en milieu nordique et [à la] gouvernance : l'exemple du parc national Tursujuq». Il est doublement intéressant puisque, d'une part, il met en évidence l'importance dans la vision autochtone du rôle d'un parc dans la protection et la mise en patrimoine de la culture. D'autre part, il montre que le processus de patrimonialisation du territoire peut s'avérer un moyen de minimiser les tensions entre les «usagers» du parc : les touristes et les Autochtones. Le territoire patrimonialisé peut donc être vu comme un médium favorisant de nouveaux modes d'interaction entre acteurs en compétition.

Le texte de Fabienne Joliet, "Ceux qui regardent font le paysage : les Inuits d'Umiujaq et le parc national Tursujuq (Nunavik)», permet quant à lui d'aborder les représentations que les jeunes Inuits ont du territoire. Cet article présente les résultats d'une recherche participative avec la communauté d'Umiujaq. Celle-ci a donné lieu à l'organisation d'un concours photographique et d'ateliers de dessin pour enfants. Les représentations du territoire produites dans le cadre de ces activités de création mettent en évidence l'importance pour les jeunes Inuits de certains lieux, notamment les chutes de la Nastapoka. Les photographies et les dessins illustrent aussi un registre de sensibilité qui est l'expression de leur vision du monde. L'échelle monumentale des paysages représentés reflète l'ancrage culturel et historique des Inuits sur ce territoire, tandis que le nombre important de scènes représentant des événements naturels tels que les couchers de soleil ou les scènes animalières révèle leur relation privilégiée à la nature. L'auteure conclut qu'il est important, dans le processus de planification des parcs, de se mettre à l'écoute de ces représentations, car elles révèlent ce qui, dans le territoire, est pour les Inuits porteur de sens, mais qui n'est pas toujours, faute de médiums, exprimé ailleurs. On pourrait aussi ajouter que de telles représentations pourraient constituer des éléments de la mise en tourisme des parcs puisque, si elles étaient partagées avec les visiteurs, elles contribueraient à leur donner accès au «monde» inuit.

Les trois derniers articles sont consacrés à la question des changements climatiques. Cette problématique peut, de prime abord, sembler assez éloignée de la question de la gouvernance des parcs ou de celle de leur potentiel touristique. À cet égard, dans une étude faite auprès d'un échantillon d'acteurs du tourisme du Nunavik, Lemelin et al. (2012) constataient que ceux-ci ne faisaient pas référence aux changements climatiques. Les auteurs en concluaient que, pour eux, «climate change may not be perceived as the most pressing source of change or disruption» (2012:52). En effet, la préoccupation principale des Autochtones en matière de tourisme et d'activités au sein des parcs concerne d'abord les répercussions des activités anthropiques sur l'écosystème et sur leurs pratiques traditionnelles. Cela dit, le changement climatique est loin d'être un acteur indifférent dans la dynamique touristique puisqu'un certain nombre d'activités, comme l'observation des ours polaires, peuvent en être directement et rapidement affectées. De plus, les voyagistes commencent à tirer parti de l'intérêt morbide de certains touristes qui souhaitent voir la banquise avant qu'elle ne disparaisse. Un voyagiste français (Terres Oubliées) utilise même la thématique de "territoire et peuple en sursis» comme argument de marketing (Antomarchi, 2009: 56).

La réflexion que proposent les articles consacrés aux changements climatiques met ainsi en évidence les enjeux et 
les possibilités qu'offrent les modèles de gouvernance novateurs pour gérer les répercussions des changements climatiques sur le territoire des parcs. Dans l'article «Cogestion adaptative des parcs du Nunavik dans un contexte de changements climatiques», Yanick Gendreau, Catherine Gagnon et Dominique Berteaux présentent le concept de cogestion adaptative et mettent en lumière, à partir d'une analyse fine de la complexité des changements climatiques anticipés au Nunavik, l'intérêt d'une telle approche parce qu'elle offre la flexibilité nécessaire pour maximiser l'implication des Autochtones. L'article de Marie-Michèle Bourassa et Philippe Auzel, «Les changements climatiques au Nunavik : de l'évaluation des impacts à une gestion intégrée des transitions pour la conservation des écosystèmes ", met l'accent sur la contribution des Autochtones à l'évaluation des impacts et à la gestion intégrée du changement climatique. Enfin, le texte de Nicolas Milot et Virginie Larivière, «La cogestion des parcs du nord du Québec en contexte d'incertitude : une occasion à saisir pour la recherche d'innovations ", s'intéresse à la gestion interculturelle et constate que la diversité culturelle et institutionnelle contribue à augmenter le potentiel d'action des acteurs de la gestion des parcs et favorise ainsi l'adaptation des mesures de protection.

Ce projet de recherche a été rendu possible grâce à une subvention du CRSH ainsi qu'au soutien financier de l'Université du Québec en Outaouais. Le directeur et rédacteur en chef de la revue, Alain A. Grenier, et moi tenons à remercier Michael Barrett, Mélanie Chabot et Mireille Boulianne de Parcs Nunavik pour leur concours exceptionnel à la réalisation de ce numéro spécial de Téoros.

Bonne lecture!

\section{Références}

ANTOMARCHI, Véronique (2009) «Tourisme, identité et développement en milieu inuit», Téoros, vol. $28, \mathrm{n}^{\circ} 1$, p. 52-60.

ANTOMARCHI, Véronique (2010) «La création du parc National des Pingualuit au Nunavik (Nord Québec), une promotion touristique révélatrice de divergences d'opinions", Cahiers de Géographie, n 10, p. 101-112.

D'AMOURS, Annie (2011) «Must We Put Dogsleds on Wheels for the Tourist Season? Inuit Heritage, Tourism and Respecting the Community in Kangiqsujuaq", dans GRENIER, Alain et Dieter MÜLLER (sous la direction de), Polar Tourism: A Tool for Regional Development, p. 109-127. Montréal : Presses de l'Université du Québec à Montréal.

GRENIER, Alain A. (2007) «The Diversity of Polar Tourism: Some Challenges Facing the Industry in Rovaniemi, Finland », Polar Geography, vol. 30, $\mathrm{n}^{\circ} 1-2$, p. 55-72.

KAUFMANN, E. et O. ZIMMER (1998) «In search of the authentic nation: landscape and national identity in Canada and Switzerland», Nations and Nationalism, vol. 4, no 4, p. 483-510.

KRG — Kativik Regional Government (2009) Position Paper on Tourism, Kuujjuaq : KRG. 8 p.

KRG — Kativik Regional Government (2007) Nunavik Tourism Trends and Opportunities, Kuujjuaq : KRG. 8 p.

LARIVIÈRE, Mylène (2010) Développement durable en territoire conventionné : le parc national Kuururjuaq, ou pragmatisme au Nunavik, texte de la communication présentée à la conférence Insight, Montréal, 28 septembre 2010, manuscrit inédit. 23 p.
LEMELIN, Raynald H.; Margaret E. JOHNSTON; Jackie DAWSON; Emma S. STEWART et Charles MATTINA (2012) «From hunting and fishing to cultural tourism and ecotourism: examining the transitioning tourism industry in Nunavik», The Polar Journal, vol. 2, n' 1, p. 39-58.

LEQUIN, Marie (2003) «Tourisme autochtone au Québec : État des lieux», Document servant de support au forum tour d'horizon sport, tourisme, culture et développement durable, Université de Nice — Sophia Antipolis, le 12 juin 2003.

LEQUIN, Marie et Isabelle CLOQUET (2006) «Facteurs sociopolitiques influent sur la gouvernance de l'offre écotouristique : Le cas des parcs nationaux au Nunavik», dans GAGNON, Christiane et Serge GAGNON (dir.), L'écotourisme, entre l'arbre et l'écorce: De la conservation au développement viable des territoires, p. 230-266. Québec: Les Presses de l'Université du Québec.

MAKIVIK (2012) Plan Nunavik, Sector by Sector Concerns and Priorities for Nunavik, Westmount : Institut culturel Avataq. 469 p.

MARTIN, Thibault (à paraître) «La place des Autochtones dans la gouvernance des parcs de l'Arctique : de la cogestion à la cojuridiction ", dans LENAERTS, Marc et Pascale VISART DE BOCARMÉ (sous la direction de), Gouvernance et cogestion au Nunavut et au Nunavik. Un état des lieux, Bruxelles : Peterlang.

MDDEP — Ministère du Développement durable, de l'Environnement et des Parcs (2005) Plan directeur. Parc national des Pingualuit, Québec: Direction du patrimoine écologique et des parcs, service des parcs. $58 \mathrm{p}$.

MDDEP - Ministère du Développement durable, de l'Environnement et des Parcs (2007a) Audience sur la création du parc national Kuururjuaq, Québec: Direction du patrimoine écologique et des parcs Service des Parcs. 37 p.

MDDEP - Ministère du Développement durable, de l'Environnement et des Parcs (2007b) Plan directeur provisoire. Projet de parc national de la Kuururjuaq, Québec: Direction du patrimoine écologique et des parcs Service des Parcs. $48 \mathrm{p}$.

MDDEP — Ministère du développement durable, de l'environnement et des parcs (2008a) «Audience publique tenue par Ministère du développement durable, de l'environnement et des parcs sur le projet de parc national des Lacs-Guillaume-Delisle-et-à-l'Eau-Claire», verbatim, MDDEP, <http://www.mddep.gouv.qc.ca/parcs/projets/Guil-Delisle Eauclaire/consultation/seances/Seance20080616.pdf >, consulté le $1^{\text {er }}$ février 2012.

MDDEP — Ministère du Développement durable, de l'Environnement et des Parcs (2008b) Plan directeur provisoire. Parc national des LacsGuillaume-Delisle-et-à-L'Eau-Claire, Québec : Direction du patrimoine écologique et des parcs, service des parcs. $47 \mathrm{p}$.

NASH, Roderick (1967) Wilderness and the American Mind, Yale (Connecticut) : Yale University Press. 288 p.

PARC NATIONAL DU CANADA DES MONTS-TORNAT (Tongait KakKasuangita SilakKijapvinga) (2010) Plan directeur, Ottawa : Agence de Parcs Canada. 65 p.

PARC NATIONAL DU CANADA QUTTINIRPAAQ (2009) Plan directeur, Ottawa : Agence de Parcs Canada. 75 p.

PARC NATIONAL DU CANADA VUNTUT (2010) Plan directeur, Ottawa : Agence de Parcs Canada. 60 p.

PARCS NUNAVIK (2012) Au sujet des parcs. Introduction, Parcs Nunavik, $<$ http://www.nunavikparks.ca/fr/introduction/presentation.htm>, consulté le 02 janvier 2013.

TULUGAK, Aliva et Peter MURDOCH (2007) A New Way of Sharing, A Personal History of the Cooperative Movement in Nunavik, Baie-d'Urfé (Québec) : Fédération des coopératives du Nouveau Québec. 287 p. 


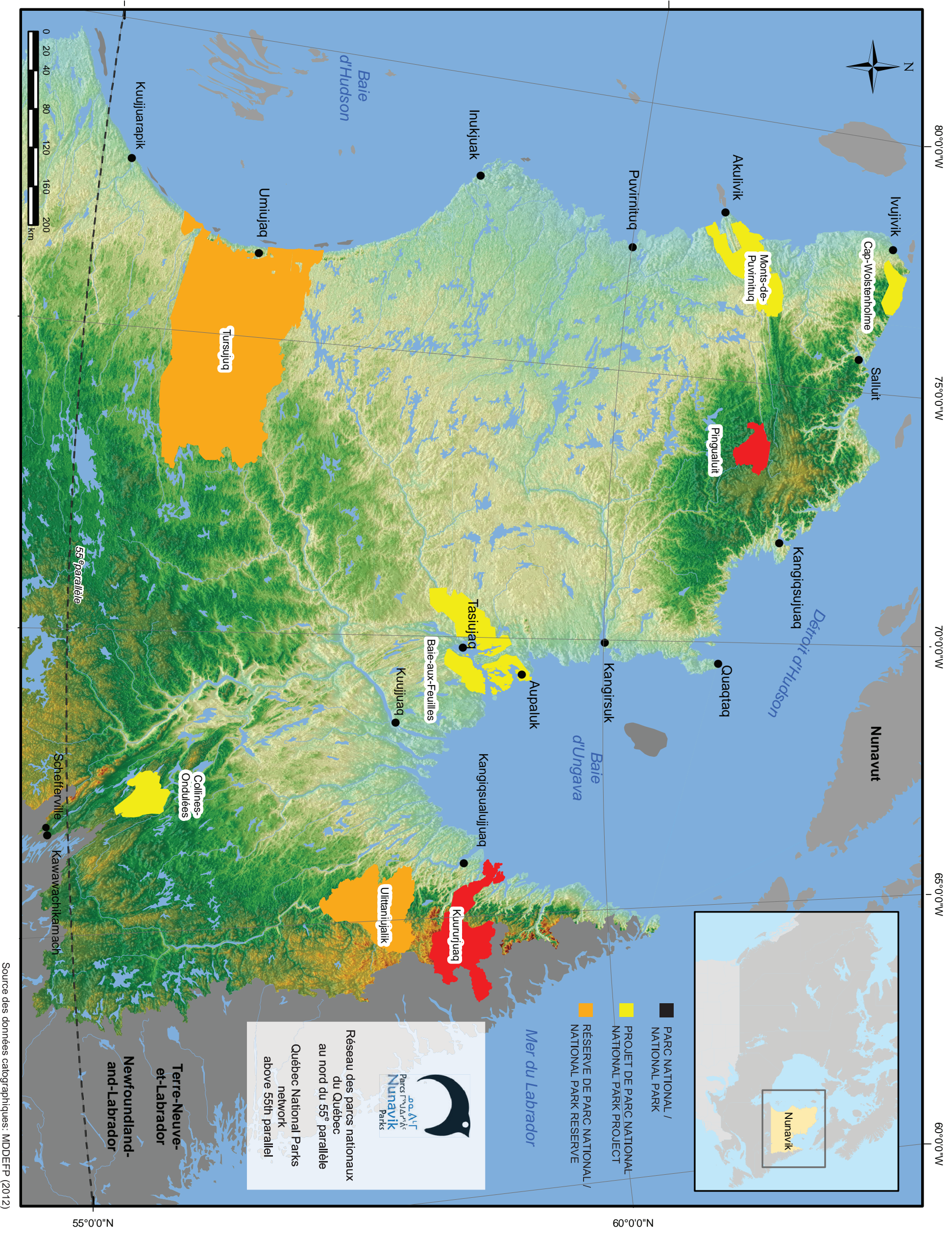

